\title{
Tunability of Triangular SRR and Wire Strip (TSRR-WS) Metamaterial at THz
}

\author{
Parul Dawar ${ }^{1}$ and Asok $\mathrm{De}^{2}$ \\ ${ }^{1}$ Department of ECE, Guru Tegh Bahadur Institute of Technology, GGSIPU, Delhi, India \\ ${ }^{2}$ NIT Patna, Bihar, India \\ Correspondence should be addressed to Asok De; asok.de@dce.edu
}

Received 19 October 2013; Revised 20 January 2014; Accepted 5 February 2014; Published 27 April 2014

Academic Editor: Augusto Beléndez

Copyright (C) 2014 P. Dawar and A. De. This is an open access article distributed under the Creative Commons Attribution License, which permits unrestricted use, distribution, and reproduction in any medium, provided the original work is properly cited.

\begin{abstract}
This paper adumbrates a novel tunable metamaterial consisting of triangular split ring resonator (TSRR) and wire strip (WS) at $\mathrm{THz}$ frequency. Ansoft high frequency structure simulator (HFSS) has been used to design and analyse the metamaterial having Rogers RT/duroid $5870\left(\varepsilon_{r}=2.33\right)$ and FR4 $\left(\varepsilon_{r}=4.4\right)$ as substrate material. Nicolson Ross Weir (NRW) method has been used to retrieve the material parameters from transmission and reflection coefficient. $4 \%$ maximization has been obtained in the location of the negative region (or resonance frequency for permeability) by using FR4 with $0.75 \mu \mathrm{m}$ instead of $1.25 \mu \mathrm{m}$ as substrate thickness. In addition, $18 \%$ minimization has been achieved by using FR4 with $0.25 \mu \mathrm{m}$ instead of RT/duroid 5870 substrate with the same thickness. Tunability has been proved by showing dependence of resonant frequency over the substrate thickness and substrate material.
\end{abstract}

\section{Introduction}

"Metamaterials" (MTMs) are engineered to modify the bulk permeability and/or permittivity of the medium. It is realized by placing periodically structures that alter the material parameters, with elements of size less than the wavelength of the incoming electromagnetic wave. It results in "meta," that is, "altered" behaviour or behaviour unattainable by natural materials. Slight changes to a repeated unit cell can be used to tune the effective bulk material properties of a MTM, replacing the need to discover suitable materials for an application with the ability to design a structure for the desired effect. Examples of MTMs are single negative materials (SNG) like $\varepsilon$ negative (ENG) which have effective negative permittivity and $\mu$ negative (MNG) which have effective negative permeability and double negative materials (DNG).

The past few years have been very eventful with respect to the evolution of the concept and implementation of "lefthanded materials (LHMs)." The cross product of $E$ and $H$ is proportional to the $k$ vector and the $E$ or $H$ field. These vectors follow the right hand rule. Power flow is described by Poynting's vector $(S)$. The material values of particular interest to the electromagnetic community are the values of permittivity, $\varepsilon$, and permeability, $\mu$. Taken together, $\varepsilon$ and $\mu$ determine the speed of electromagnetic propagation through a medium and the square root of their product determines the refractive index $(n)$. A real wave vector indicates a propagating wave, while an imaginary wave vector indicates attenuation (an evanescent wave). Therefore, upon entering a medium with altered material parameters, that is, negative $\varepsilon$ and $\mu$, the group and phase velocities have opposite signs and are antiparallel, indicating that wave fronts move towards a source in this material creating a "backwards wave." However, Poynting's vector, which is defined by $E \times H$, is still positive, power travels away from the source, and causality is maintained. Thus the $S$ vector follows the right hand rule, while the $k$ vector is antiparallel to the $S$ vector. It can be illustrated as in Figure 1.

Table 1 depicts the sign rules for material refractive index while Table 2 introduces some basic properties and names for materials for various combinations of permeability and permittivity.

A fresh approach to microwave and optical devices presented itself with the interesting breakthrough in the area of MTMs at high frequencies. The need of hour is to optimize 

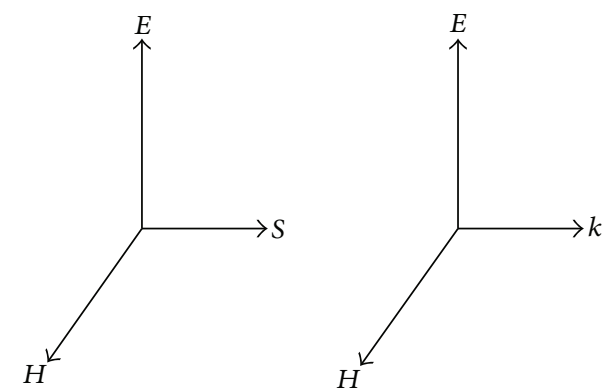

(a)

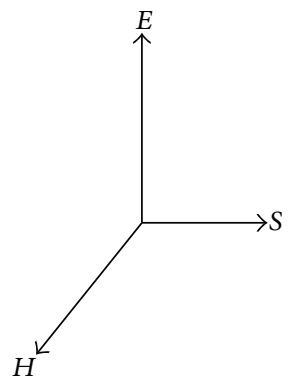

(b)

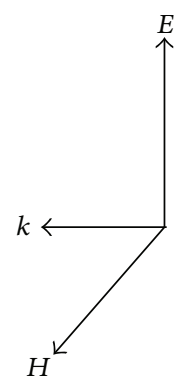

FIGURE 1: The wave propagation in a (a) right-handed and (b) left-handed system.

TABLE 1: Sign rules of the refraction index.

\begin{tabular}{lcc}
\hline & $\varepsilon>0$ & $\varepsilon<0$ \\
\hline$\mu>0$ & Positive & Imaginary \\
$\mu<0$ & Imaginary & Negative \\
\hline
\end{tabular}

the antenna parameters (gain, bandwidth, and directivity) without altering its dimensions, that is, external control over antenna parameters using MTM. The software tool high frequency structure simulator (HFSS) is used because it is a high performance full wave electromagnetic (EM) field simulator for arbitrary $3 \mathrm{D}$ volumetric passive device modeling. It integrates simulation, visualization, solid modeling, and automation in an easy way to learn environment where solutions to 3D EM problems are quickly and accurately obtained [1].

Section 2 reviews metamaterial in high frequency including $\mathrm{THz}$ regime. Section 3 abridges the design of metamaterial composed of triangular SRR and wire strip. Section 4 illustrates the tunability of the metamaterial with varying substrate thickness and substrate material. Section 5 concludes the paper.

\section{Metamaterials at High Frequency (including THz Regime)}

The terahertz region of the electromagnetic spectrum can be defined as occupying the high frequency range $0.1-10 \mathrm{THz}$ and bounding the microwave and midinfrared regions. In the last twenty years, a lot of applications implementing the "terahertz gap" have been seen.

Demonstration of LH behaviour was done by two designs: the first is SRR with wire structures and the second is closed SRR with wire structures [2] as in Figure 2(a). The measured transmission spectrum gives first band gap $(3.55-4.0 \mathrm{GHz})$ in SRR, not in CSRR medium. It states that stop band of SRR medium cannot be automatically attributed to negative $\mu$ behaviour; some observed gaps could also originate from the electrical response of SRRs or from Bragg gaps due to periodicity. Also, it proves that SRRs contribute to effective permittivity of composite MTM (CMM), causing downward shift on the plasma frequency determined solely from wire structures. It was also observed that increasing the number of layers decreased the phase of transmitted EM wave at LH frequency region.

Another demonstration of negative permittivity of MTMs was done by a structural unit cell of proposed electric disk resonator which consisted of two disks connected by a central post as in Figure 2(b). Using Ampere's circuital law, we can relate flux on disk faces and the resonant behaviour of EDR unit cell. Above the resonant frequency of unit cell, effective permittivity is negative, where the electric resonance frequency is independent on the leakage capacitance between plates and post. It was observed that electric field reverses direction just above or just below the resonant frequency of unit cell, thus depicting negative $\varepsilon$ values by Ansoft high frequency structure simulator.

MTM sample consisting of hexaferrite slab and metallic array of parallel wires ensures the corresponding negative values for the effective permeability in a frequency domain which overlaps the domain with negative permittivity [3] as in Figure 2(c). An expression of effective permittivity tensor states that subdomain of negative permittivity values can be controlled by varying some parameters in a finite range. It has been observed that for thinner wires the permittivity maximum shifts to higher frequencies, but permittivity magnitude decreases.

Grbic [4] demonstrated a 2D composite medium that has broadband negative permeability and negative permittivity as in Figure 2(d). The medium was isotropic and exhibited LH behaviour over $60 \%$ bandwidth. Broadband negative permeability medium employed embedded resonant elements (miniaturized SRR) tuned to different frequencies and separated by layers of impedance inverters. SRRs, square shaped, with metallic strips loaded with lumped element capacitors, having a vertical wire, reacted to $z$ directed electric field at centre of unit cell and yielding $\varepsilon$ negative. Ansoft HFSS was used to infer $60 \%$ bandwidth of LH behaviour with centre frequency where the left-handed band (backward wave) intersects the light line.

In references [5-9], all papers were abridging microwave frequencies (X-band or below). The first electromagnetic metamaterial produced by microfabrication has been reported [10]. By reducing the overall rod-SRR structural size below $100 \mu \mathrm{m}$ and structural details down to $5 \mu \mathrm{m}$, 

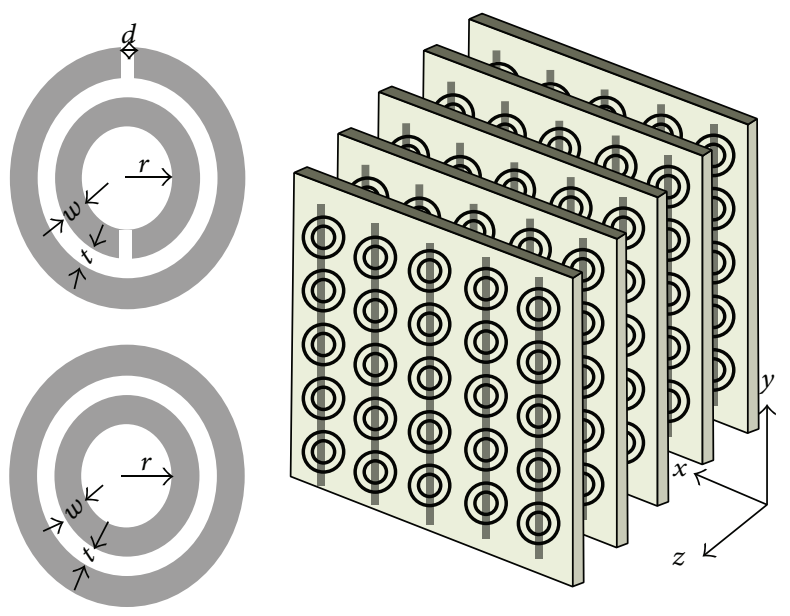

(a)

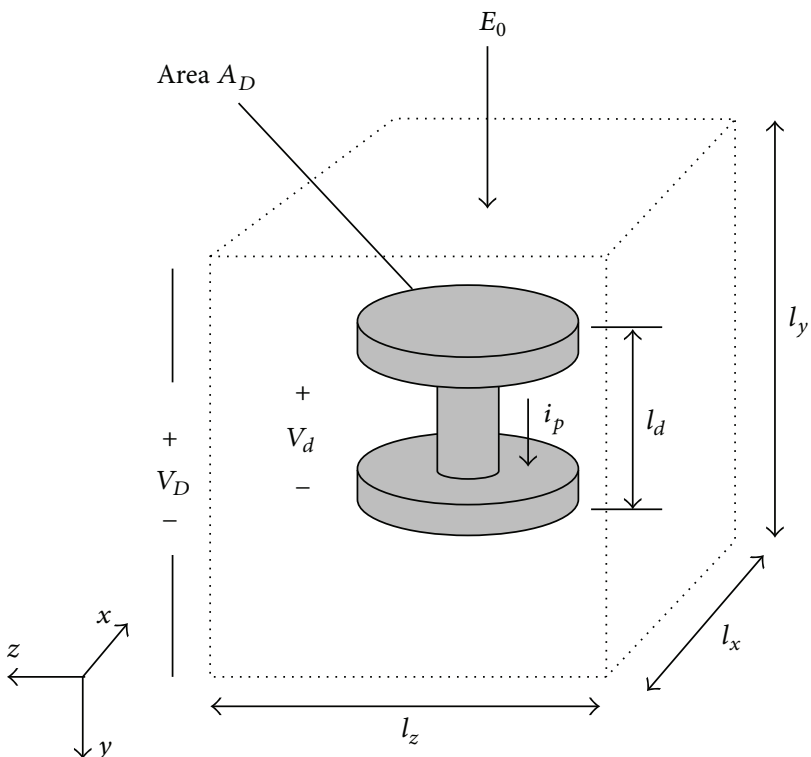

(b)

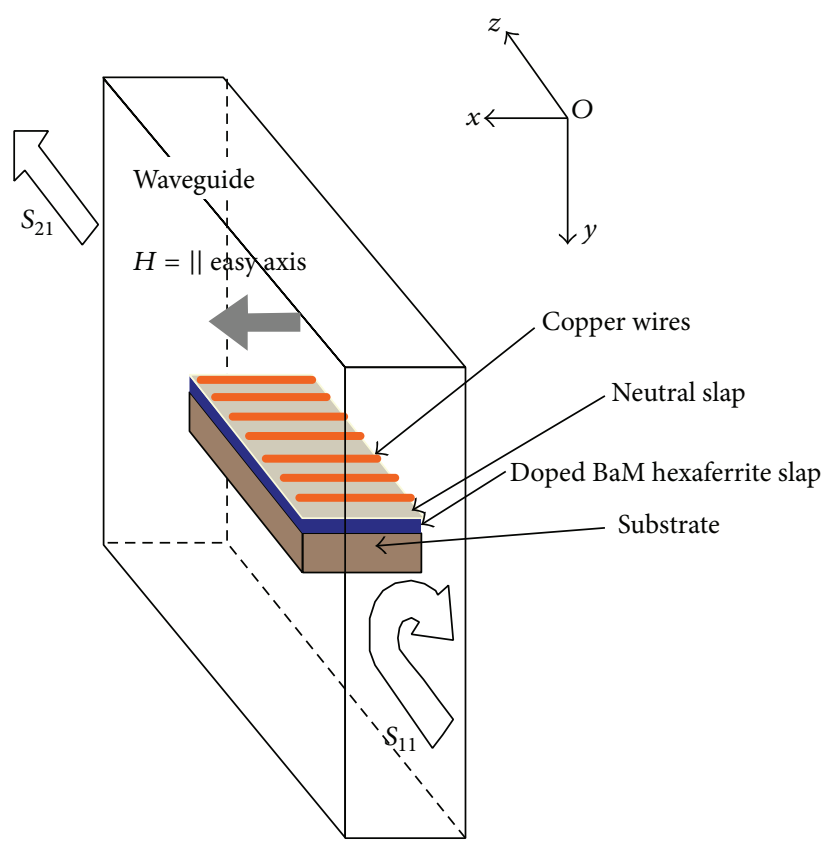

(c)

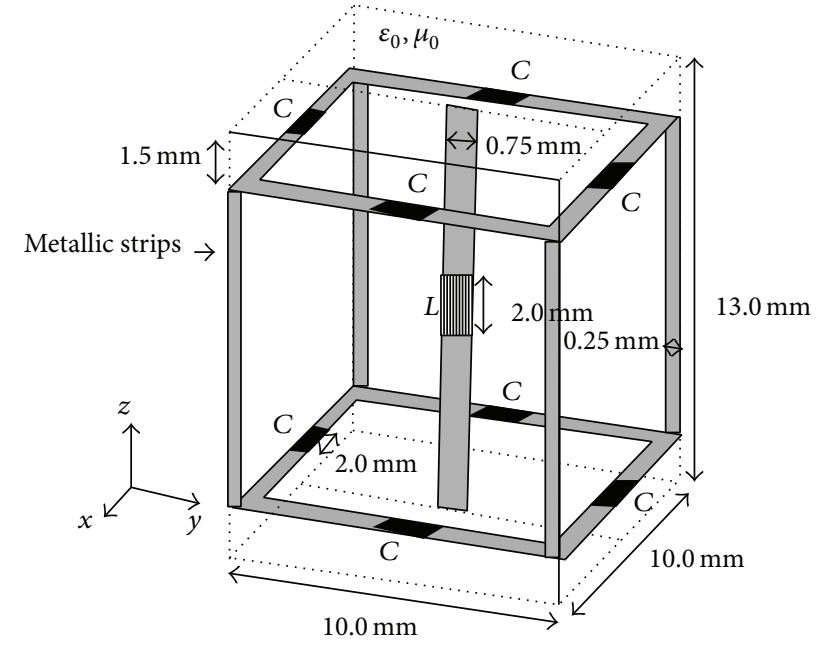

(d)

FIGURE 2: (a) The first is SRR with wire structures and the second is closed SRR with wire structures, (b) electric disk resonator, (c) hexaferrite slab with metallic array of parallel wires, and (d) 2D composite medium.

TABLE 2: Microwave permittivity and permeability characteristics of homogeneous metamaterials.

\begin{tabular}{lccc}
\hline$\varepsilon>0, \mu>0$ & $\varepsilon<0, \mu>0$ & $\varepsilon>0, \mu<0$ & $\varepsilon<0, \mu<0$ \\
\hline \multirow{3}{*}{ Forward wave propagation } & (i) Metal-like characteristics at & (i) Ferrimagnetic material & (i) Left-handed medium \\
& optical frequencies & characteristics & (ii) Backward-wave propagation \\
& (ii) Evanescent waves & (ii) Evanescent waves & (iii) Re $(n)<0$
\end{tabular}


the frequency at which composite material exhibits the electromagnetic MTM behaviour increased (to few THz). The design data of structure was determined by using Pendry's [7] analytical formulas as well as numerical simulation by means of microwave studio. Five geometric variants were considered by changing $r, d, c, l$. This structure has EM properties in $1-2.7 \mathrm{THz}$ range. Thus, the spectral range has been extended by three orders of magnitude.

Two different planar MTM consisting of unit cell of SRR and eSRR were fabricated on intrinsic GaAs substrate [11]. This achieved active and dynamic control over MTM at $\mathrm{THz}$ frequencies by dynamically switchable THz MTMs. Using ErAs/GaAs nano island super lattice very short carrier lifetime and ultra fast switching recovery of MTM resonance was achieved. All electronically switchable THz MTM devices are hybrid of schottky diode structure and eSRR array, where it is necessary to connect all eSRRs together serving as schottky contact. It proved that there is neither a shift of resonance frequency nor a change of resonance strength of eSRR by connecting wires. Photo excitation was used to change the $\varepsilon$ values (real), at resonance. However, strong resonance dip is observed with no photo excitation.

Six original MTM unit cells (OE1-OE6) and their complements (CE1-CE6) which are planar electric split ring resonator (eSRR) MTMs and their corresponding inverse structures [4] have been designed, modeled, and characterized as shown in Figure 3(a), which are resonant at $\mathrm{THz}$ frequencies. Similar to Babinet's principle, their complementary transmission properties related to that obtained by $\mathrm{THz}$ time-domain spectroscopy (THz-TDS). It illustrated that Drude and Lorentz responses lead to the transmission maximum of complementary MTM and second transmission minimum (OE) or maximum (CE) was a result of excitation of electric dipoles. Various types of subwavelength resonators, for example, thin wires, swiss rolls, SRRs, pair of rods, pair of crosses, and eSRRs [12], have also been observed as shown in Figures 3(b)-3(d).

It has been proved experimentally that planar metamaterial slab can act as a waveguide in $\mathrm{THz}$ frequency region, that is, split ring resonator structure, thereby depicting strong Lorentz-type resonances at desired frequencies [13]. Any waveguide mode can be excited with THz TD-ATR spectroscopy. Here through the ATR prism an evanescent field was generated, which interacted with the sample, and thereby an eigen mode was excited, provided its wave number equalled that of the evanescent field. It showed that where dispersion curves of waveguide modes and evanescent field cross, a waveguide mode is excited.

\section{Proposed MTM}

Tunable metamaterial has been proposed having electric and magnetic resonance $[14,15]$. It behaves as DNG, that is, double negative group. Such materials have negative permeability and negative permittivity over some frequency region [16]. The parameter retrieval, that is, parameter extraction using $S$ parameters [9], has been followed using NRW approach to observe the negative permeability region of SRR MTM.
3.1. Constructional Details. It consists of triangular SRR and wire strip. Figure 4 shows the geometry of unit cell having TSRR on one face and WS on the other face of the substrate material. TSRR and WS are made up of copper having conductivity $5.8 * 10^{-7} \mathrm{~S} / \mathrm{m}$ and having thickness of $35 \mathrm{~nm}$. The WS runs throughout the substrate having strip width of $0.5 \mu \mathrm{m}$. The width of TSRR is $0.3 \mu \mathrm{m}$ and the spacing between two TSSRs is $0.4 \mu \mathrm{m}$. Base of triangle is $7.794 \mu \mathrm{m}$ and height of triangle is $6.75 \mu \mathrm{m}$. Two types of materials are used as substrate, FR4 (with relative permittivity $\varepsilon_{r}=4.4$ and loss tangent $\pm=0: 02$ ) and Rogers RT/duroid 5880 (with relative permittivity $\left.\varepsilon_{r}=2.33\right)$. SRRs are metallic rings made of metal with a split introduced in its structure. When a current circulates a coil, it creates a magnetic dipole moment. The generated dipole moment vector is at right angles to the plane of the coil. The coil with a plate capacitor together gives us an LC circuit and therefore an increased dipole moment at its resonance.

Therefore, this structure can be considered as resonant LC type structure with resonant frequency $\omega_{r}$ given by

$$
\omega_{r}=\sqrt{\frac{1}{L \cdot C}},
$$

where the capacitance $(C)$ in the conventional nested SRR based MTMs is formed by edge coupling between the inner and outer ring. When the thickness of the substrate is decreased, the capacitance will increase, and thus the resonance frequency will decrease.

3.2. Simulation Results. Ansoft HFSS has been used to simulate the unit cell designed in Figure 5(a) having metamaterial in the dielectric substrate bounded by box on either side having air as material and radiation boundary. Open, electric, magnetic, and periodic boundary conditions are used in the simulation. Each configuration is placed in a two-port waveguide formed by a pair of both perfect electric conductor (PEC) and perfect magnetic conductor (PMC) walls. All FR4 substrates with TSRR and WS are centered in the waveguide. The boundaries and lumped ports (1 and 2) have been assigned as per Figures 5(b), 5(c), and 5(d). Nicolson Ross Weir (NRW) method has been used to calculate the material properties from transmission and reflection coefficients. The NRW [11] approach begins by introducing the composite terms,

$$
\begin{aligned}
& V 1=S 21-S 11, \\
& V 2=S 21-S 11 .
\end{aligned}
$$

Then, calculate permittivity and permeability by

$$
\begin{aligned}
& \mu_{r}=\frac{2}{j k_{o} d} \frac{1-V 2}{1+V 2}, \\
& \varepsilon_{r}=\frac{2}{j k_{o} d} \frac{1-V 1}{1+V 1} .
\end{aligned}
$$




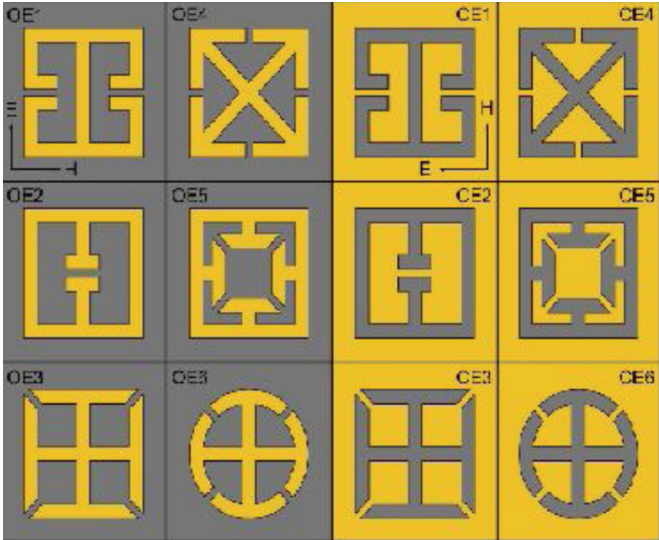

(a)

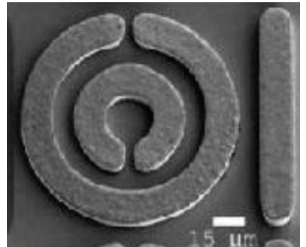

(b)

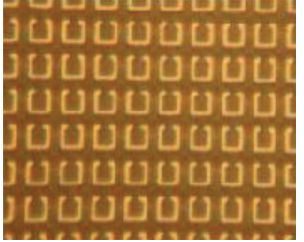

(d)

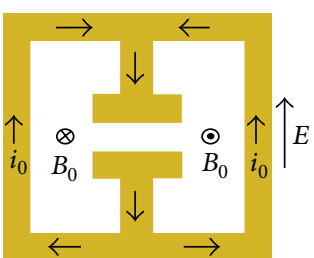

(c)

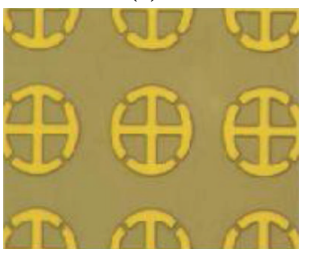

(e)

FIgURE 3: (a) Geometry of original planar metamaterial unit cells (OE1-OE6) and their complements (CE1-CE6) (b) NIM from a ring-wire structure. (c) eSRRs (d) Array of SRRs (e) Polarization-invariant eSRRs.

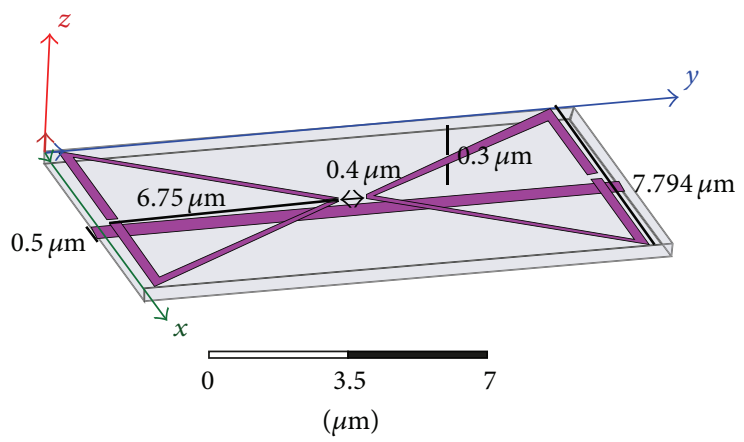

FIGURE 4: TSRR and WS MTM: unit cell designed in HFSS.

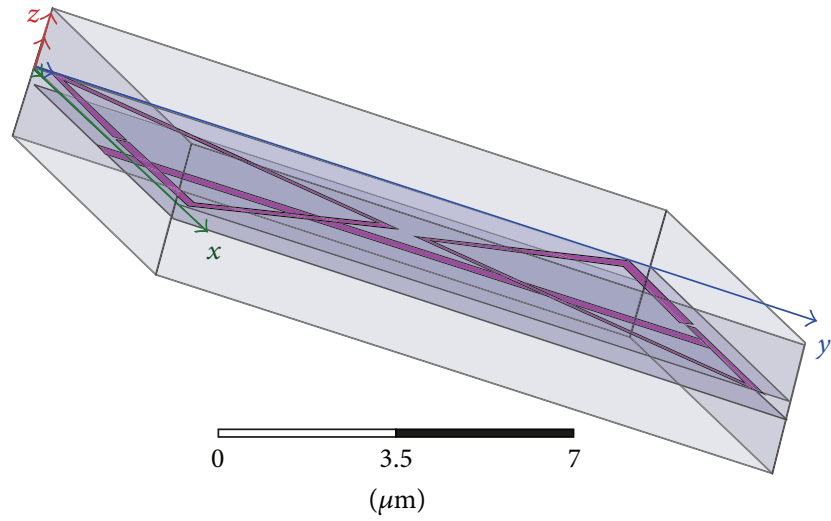

(a)

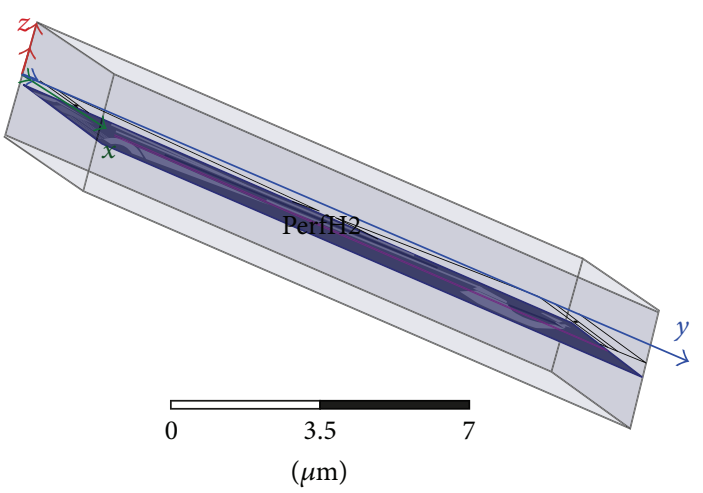

(b) H Field

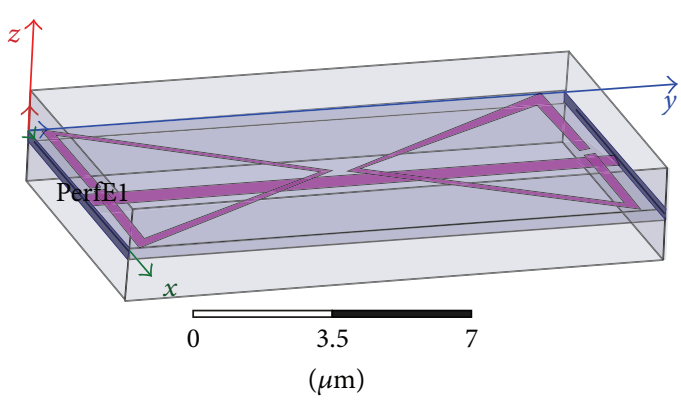

(c) E Field

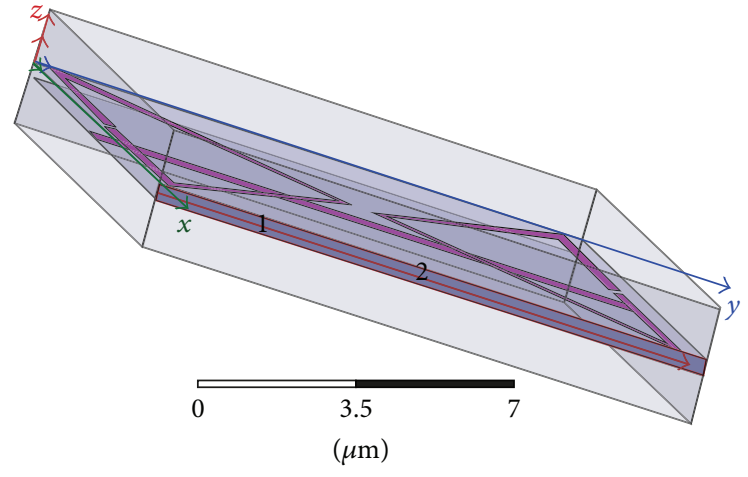

(d) Lumped port

FIgURE 5: (a) Unit cell for simulation; (b) $H$ field (c); $E$ field specifying boundary condition; (d) lumped port. 


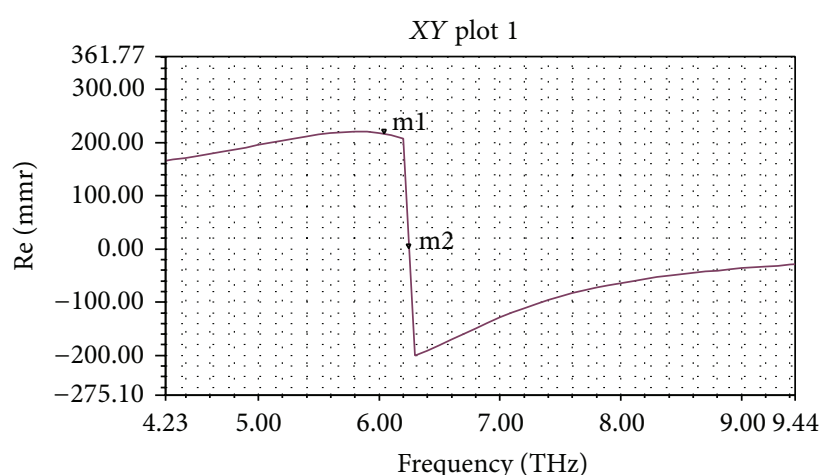

- Re (mmr)

Setup1: sweep

\begin{tabular}{|c|c|c|}
\hline Name & $X$ & $Y$ \\
\hline $\mathrm{m} 1$ & 6.0505 & 215.3136 \\
\hline $\mathrm{m} 2$ & 6.2510 & -0.2410 \\
\hline
\end{tabular}

(a)

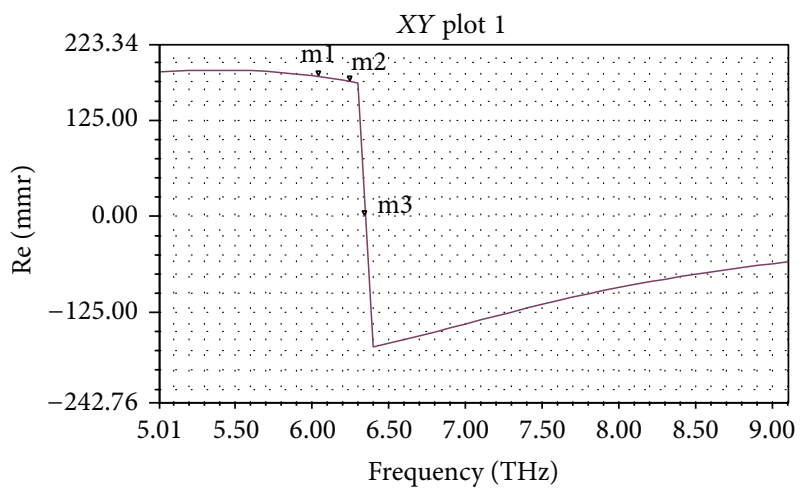

- Re (mmr)

Setup1: sweep

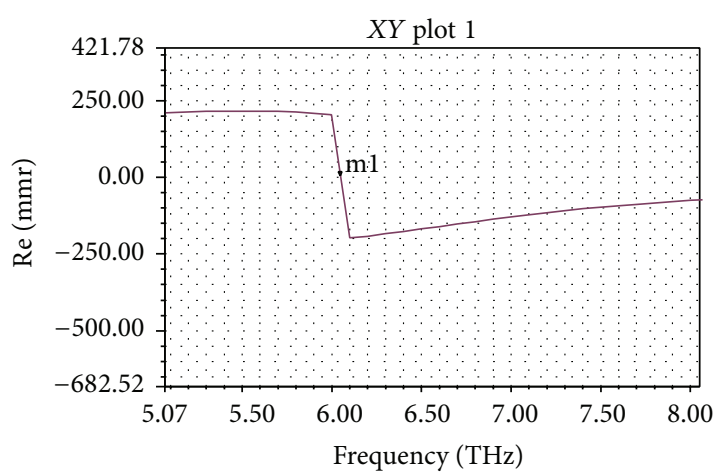

- $\operatorname{Re}(\mathrm{mmr})$ Setup1: sweep

(b)

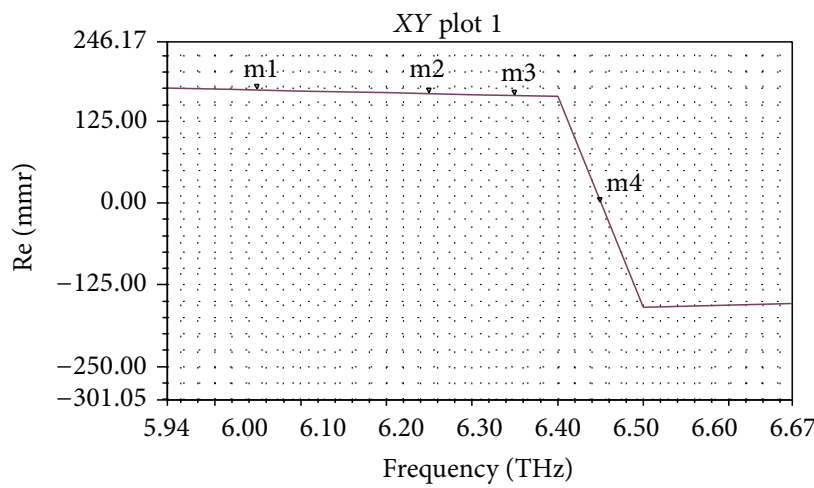

- Re (mmr) Setup1: sweep

\begin{tabular}{|c|c|c|}
\hline Name & $X$ & $Y$ \\
\hline $\mathrm{m} 1$ & 6.0505 & 181.4075 \\
\hline $\mathrm{m} 2$ & 6.2510 & 175.0585 \\
\hline $\mathrm{m} 3$ & 6.3504 & 0.7952 \\
\hline
\end{tabular}

(c)

FiguRE 6: Variations in resonance of permeability with substrate thicknesses (a) $0.25 \mu \mathrm{m}$, (b) $0.5 \mu \mathrm{m}$, (c) $0.75 \mu \mathrm{m}$, and (d) $1.25 \mu \mathrm{m}$ with RT duroid substrate.

\section{Tunability}

Tunability has been achieved by changing the thickness of the substrate and checking its effect on the resonant frequency of permeability of the MTM and plasma frequency of permittivity of the MTM. This will help us understand how the resonant frequency and plasma frequency shift by varying the substrate thickness as $0.25 \mu \mathrm{m}, 0.5 \mu \mathrm{m}, 0.75 \mu \mathrm{m}$, and $1.25 \mu \mathrm{m}$. This study has been performed first by taking RT duroid as substrate and then FR4 as substrate. Figures $6(\mathrm{a}), 6(\mathrm{~b}), 6(\mathrm{c})$, and $6(\mathrm{~d})$ give the variations in resonant frequency of permeability and Figures $7(\mathrm{a})-7(\mathrm{~d})$ give the variations in plasma frequency of permittivity with RT duroid as substrate $\left(\varepsilon_{r}=2.33\right)$. Table 3 gives the variations of plasma frequency of permittivity and resonant frequency of permeability for varying substrate thickness for RT duroid substrate.
TABLE 3: Variations of resonant frequency of permeability and plasma frequency of permittivity with substrate thicknesses for RT duroid substrate.

\begin{tabular}{lcc}
\hline $\begin{array}{l}\text { Substrate thickness } \\
(\mu \mathrm{m})\end{array}$ & $\begin{array}{c}\text { Resonant frequency } \\
(\mathrm{THz})\end{array}$ & $\begin{array}{c}\text { Plasma frequency } \\
(\mathrm{THz})\end{array}$ \\
\hline 0.25 & 6.0505 & 6.0505 \\
0.5 & 6.25 & 6.2489 \\
0.75 & 6.3504 & 6.3496 \\
1.25 & 6.4503 & 6.4501 \\
\hline
\end{tabular}

Curves in Figures 8 and 9 show that both the resonance in permeability and plasma frequency for permittivity increase nonlinearly with increase in substrate thickness.

Next study has been performed by taking FR4 as substrate. Figures 10(a), 10(b), 10(c), and 10(d) give the variations 

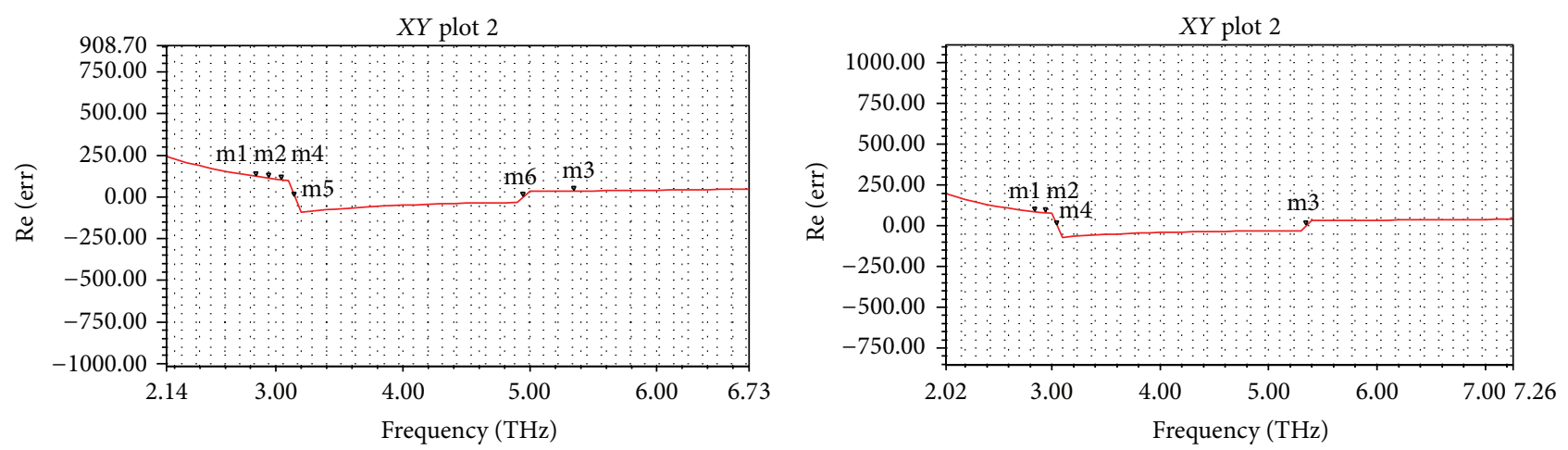

\begin{tabular}{l|c|c|c|}
\hline Re (err) & Name & $X$ & $Y$ \\
\hline Setup1: sweep & $\mathrm{m} 1$ & 2.8523 & 122.7899 \\
\hline $\mathrm{m} 2$ & 2.9513 & 112.5044 \\
\hline $\mathrm{m} 3$ & 5.3498 & 35.0886 \\
\hline $\mathrm{m} 4$ & 3.0517 & 103.1129 \\
\hline $\mathrm{m} 5$ & 3.1530 & -1.6342 \\
\hline $\mathrm{m} 6$ & 4.9500 & -0.1157 \\
\hline
\end{tabular}

\begin{tabular}{|c|c|c|c|}
\hline \multirow{5}{*}{$\begin{array}{l}\text { Re (err) } \\
\text { Setup1: sweep }\end{array}$} & Name & $X$ & $Y$ \\
\hline & $\mathrm{m} 1$ & 2.8523 & 86.6334 \\
\hline & $\mathrm{m} 2$ & 2.9513 & 79.7964 \\
\hline & $\mathrm{m} 3$ & 5.3498 & -0.0593 \\
\hline & $\mathrm{m} 4$ & 3.0517 & 0.3985 \\
\hline
\end{tabular}

(a)

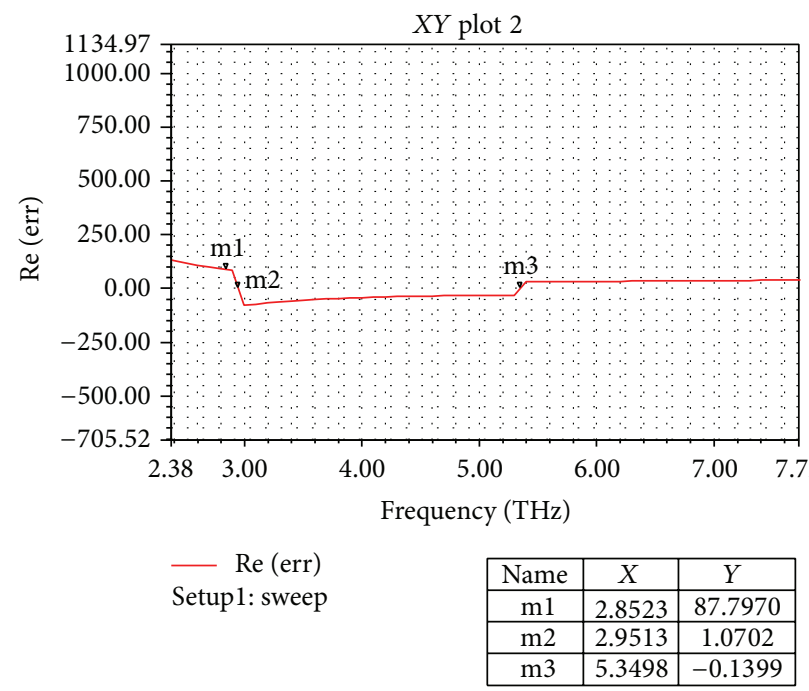

(b)

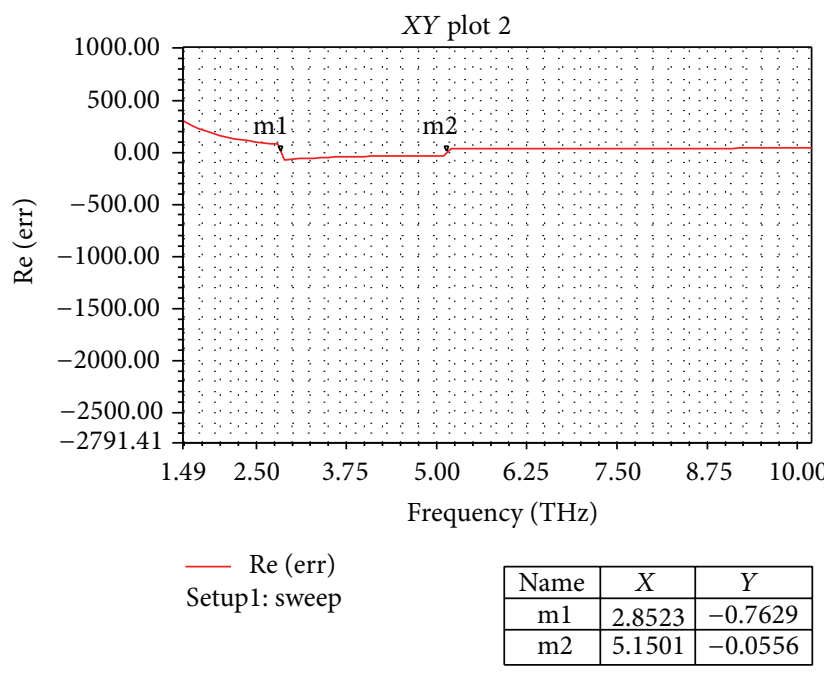

(c)

(d)

FiguRE 7: Variations in plasma frequency of permittivity with substrate thicknesses (a) $0.25 \mu \mathrm{m}$, (b) $0.5 \mu \mathrm{m}$, (c) $0.75 \mu \mathrm{m}$, and (d) $1.25 \mu \mathrm{m}$ with RT duroid substrate.

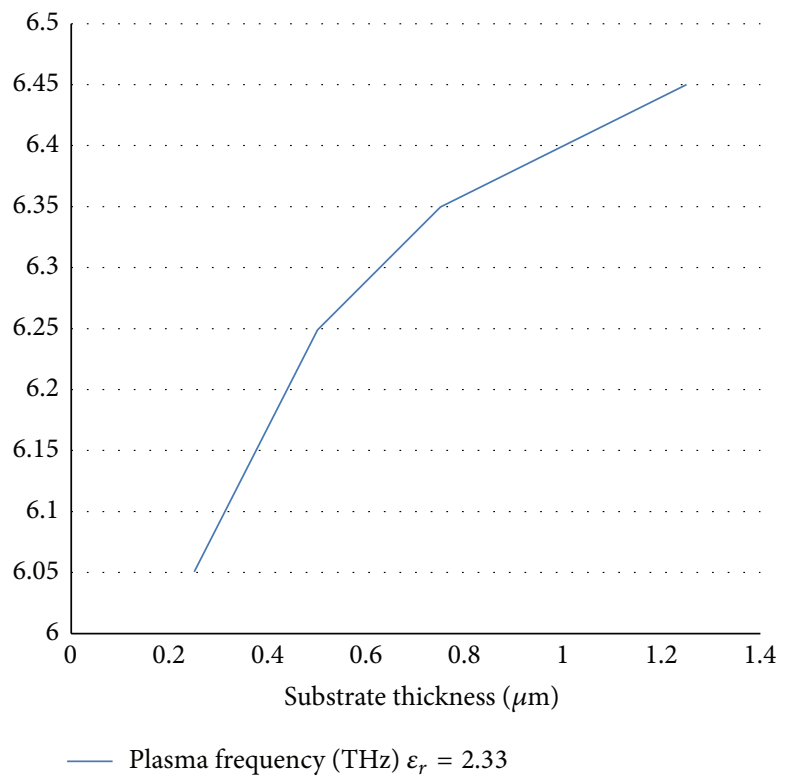

FIGURE 8: Curve showing variations of plasma frequency with substrate thickness for RT duroid substrate. 


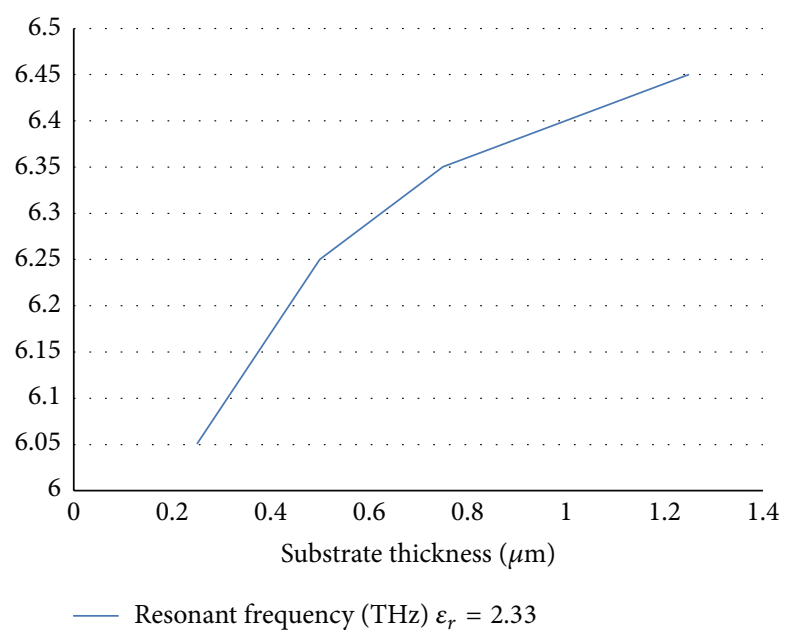

FIGURE 9: Curve showing variations of resonance in permeability with substrate thickness for RT duroid substrate.

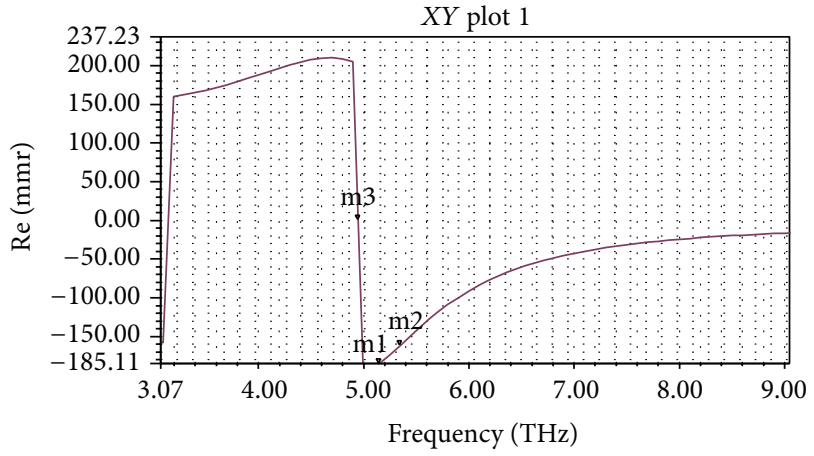

- Re (mmr) Setup1: sweep

\begin{tabular}{|c|c|c|}
\hline Name & $X$ & $Y$ \\
\hline $\mathrm{m} 1$ & 5.1515 & -184.4348 \\
\hline $\mathrm{m} 2$ & 5.3489 & -161.3862 \\
\hline $\mathrm{m} 3$ & 4.9508 & 0.0954 \\
\hline
\end{tabular}

(a)

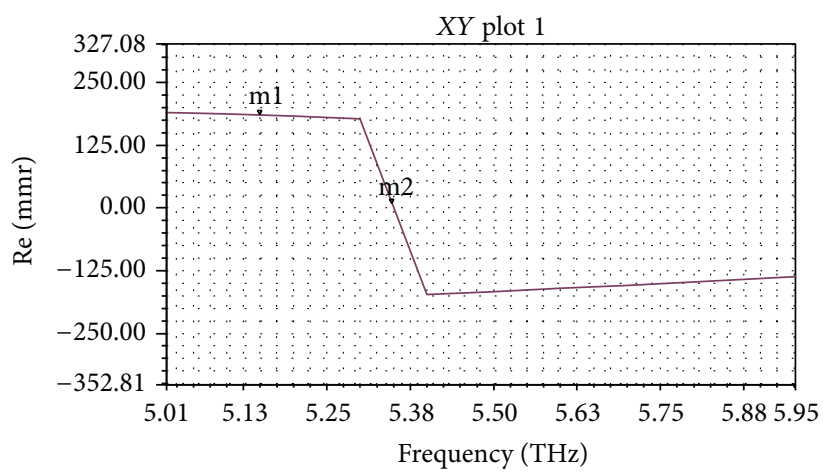

— Re (mmr)

Setup1: sweep

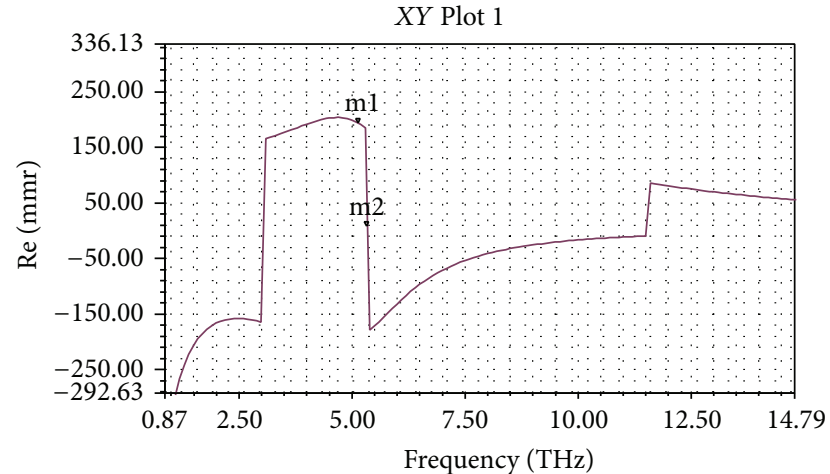

— Re (mmr)

Setup1: sweep

\begin{tabular}{|c|c|c|}
\hline Name & $X$ & $Y$ \\
\hline $\mathrm{m} 1$ & 5.1515 & 192.9400 \\
\hline $\mathrm{m} 2$ & 5.3489 & 7.3701 \\
\hline
\end{tabular}

(b)

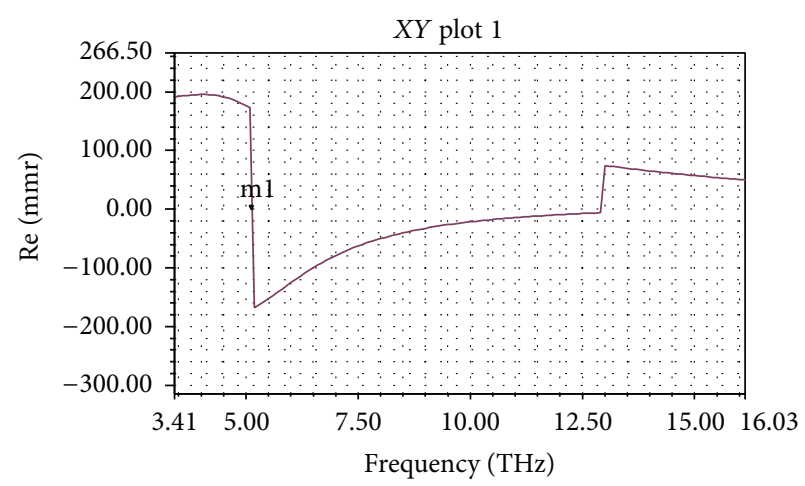

— Re (mmr)

Setup1: sweep

\begin{tabular}{|c|c|c|}
\hline Name & $X$ & $Y$ \\
\hline $\mathrm{m} 1$ & 5.1515 & -2.7798 \\
\hline
\end{tabular}

(d)

(c)

\begin{tabular}{|c|c|c|}
\hline Name & $X$ & $Y$ \\
\hline $\mathrm{m} 1$ & 5.1515 & 184.3545 \\
\hline $\mathrm{m} 2$ & 5.3489 & 6.6382 \\
\hline
\end{tabular}

FIGURE 10: Variations in resonance of permeability with substrate thicknesses (a) $0.25 \mu \mathrm{m}$, (b) $0.5 \mu \mathrm{m}$, (c) $0.75 \mu \mathrm{m}$, and (d) $1.25 \mu \mathrm{m}$ with FR4 substrate. 

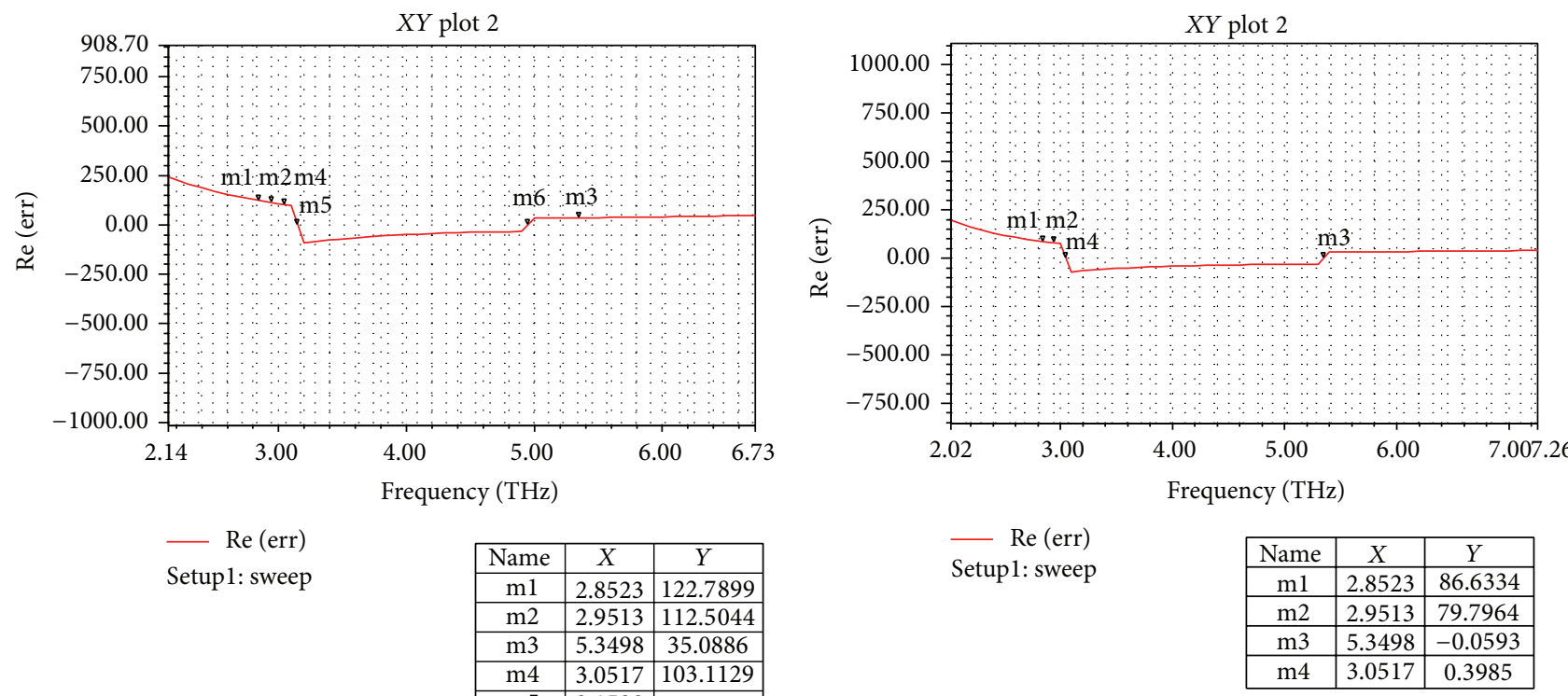

\begin{tabular}{|c|c|c|c|}
\hline \multirow{7}{*}{$\begin{array}{l}\mp \operatorname{Re}(\mathrm{err}) \\
\text { Setup1: sweep }\end{array}$} & Name & $X$ & $Y$ \\
\hline & $\mathrm{ml}$ & 2.8523 & 122.7899 \\
\hline & $\mathrm{m} 2$ & 2.9513 & 112.5044 \\
\hline & $\mathrm{m} 3$ & 5.3498 & 35.0886 \\
\hline & $\mathrm{m} 4$ & 3.0517 & 103.1129 \\
\hline & m5 & 3.1530 & -1.6342 \\
\hline & m6 & 4.9500 & -0.1157 \\
\hline
\end{tabular}

(a)

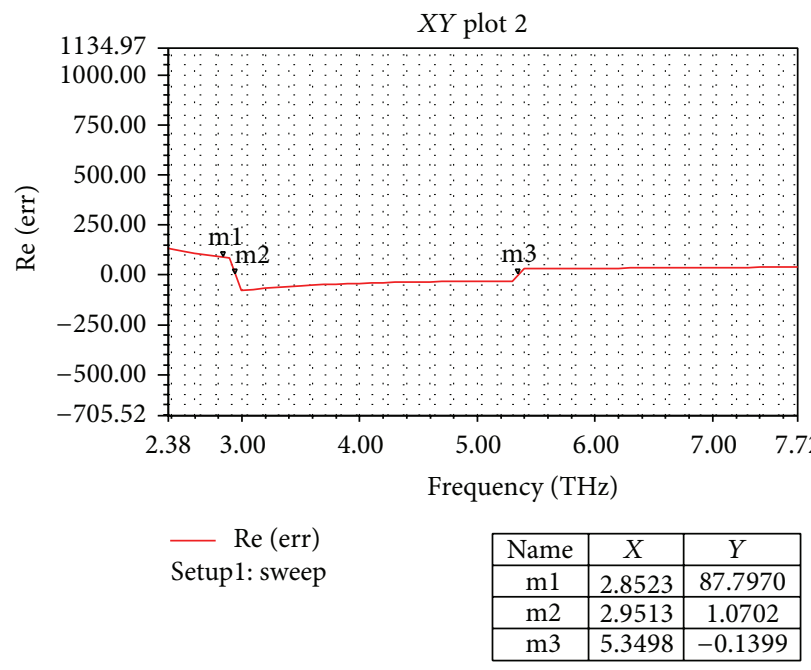

(c)

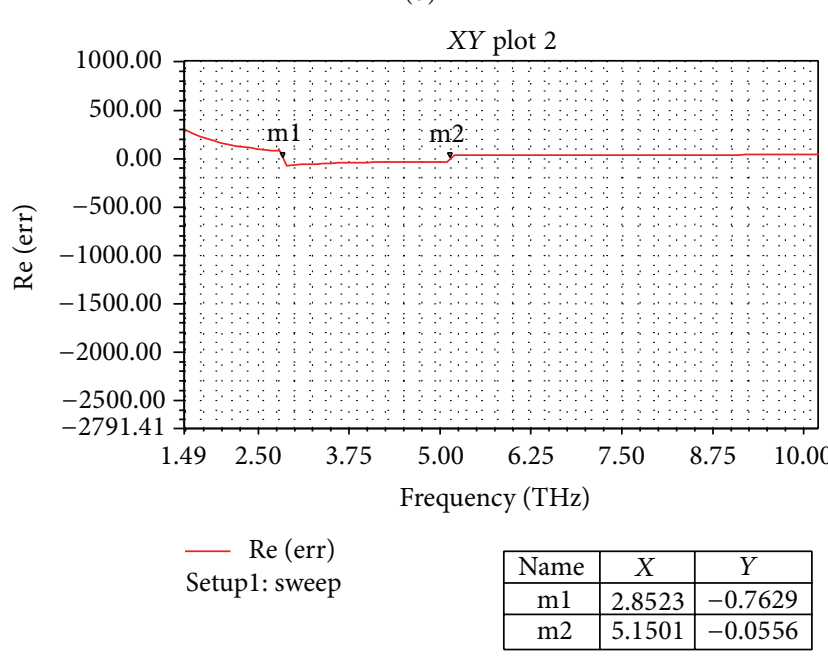

(d)

FIGURE 11: Variations in plasma frequency of permittivity with substrate thicknesses (a) $0.25 \mu \mathrm{m}$, (b) $0.5 \mu \mathrm{m}$, (c) $0.75 \mu \mathrm{m}$, and (d) $1.25 \mu \mathrm{m}$ with FR4 substrate.

in resonant frequency of permeability and Figures 11(a)-11(d) give the variations in plasma frequency of permittivity with FR4 as substrate $\left(\varepsilon_{r}=4.4\right)$. Table 4 gives the variations of plasma frequency of permittivity and resonant frequency of permeability for varying substrate thickness for FR4 as substrate.

Curve in Figure 12 shows that both the resonance in permeability and plasma frequency for permittivity vary nonlinearly with increase in substrate thickness.

It is obvious that one can easily obtain minimization or maximization in the frequency shifting as desired/required amount by tuning the substrate thickness and also by changing the material of the substrate used.

We conclude that $4 \%$ maximization has been obtained in the location of the negative region (or resonance frequency
TABLE 4: Variations of resonant frequency of permeability and plasma frequency of permittivity with substrate thicknesses for FR4 substrate.

\begin{tabular}{lcc}
\hline $\begin{array}{l}\text { Substrate thickness } \\
(\mu \mathrm{m})\end{array}$ & $\begin{array}{c}\text { Resonant frequency } \\
(\mathrm{THz})\end{array}$ & $\begin{array}{c}\text { Plasma frequency } \\
(\mathrm{THz})\end{array}$ \\
\hline 0.25 & 4.9508 & 4.95 \\
0.5 & 5.3551 & 5.3489 \\
0.75 & 5.3489 & 5.3498 \\
1.25 & 5.1515 & 5.1501 \\
\hline
\end{tabular}

for permeability) by using FR4 with $0.75 \mu \mathrm{m}$ instead of $1.25 \mu \mathrm{m}$. In addition, $18 \%$ minimization has been achieved by 


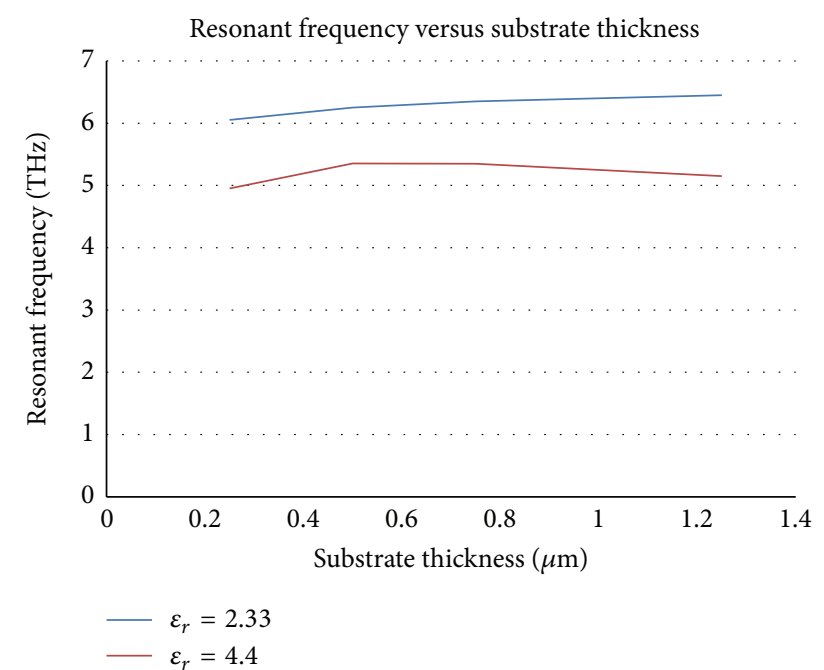

FIGURE 12: Curve showing variations of resonance frequency with substrate thickness for RT duroid substrate and FR4 substrate.

using FR4 with $0.25 \mu \mathrm{m}$ instead of RT/duroid 5880 substrate with the same thickness.

\section{Conclusion}

In this paper, a novel metamaterial, triangular SRR with wire strip, has been designed focusing on the high frequency $(\mathrm{THz})$. Also, metamaterial design at high frequency has been reviewed with various different metallic structures, forming unit cells. We conclude that minimization or maximization can be obtained in the frequency shifting as desired/required amount by tuning the substrate thickness and also by changing the material of the substrate used. We conclude that $4 \%$ maximization has been obtained in the location of the negative region (or resonance frequency for permeability) by using FR4 with $0.75 \mu \mathrm{m}$ instead of $1.25 \mu \mathrm{m}$. In addition, $18 \%$ minimization has been achieved by using FR4 with $0.25 \mu \mathrm{m}$ instead of RT/duroid 5880 substrate with the same thickness.

The exigence of metamaterials as substrate or superstrates in antenna can be seen with various improvements in optimization of its parameters, like gain, bandwidth, side lobes, directivity, and size in near future.

\section{Conflict of Interests}

The authors declare that there is no conflict of interests regarding the publication of this paper.

\section{References}

[1] http://www.docstoc.com/docs/127876758/High-FrequencyStructure-Simulator-_HFSS_-Tutorial.

[2] E. Ozbay and C. M. Soukoulis, "Observation of negative refraction and negative phase velocity in true left-handed metamaterials," in Proceedings of the 36th European Microwave Conference (EuMC '06), pp. 959-962, September 2006.
[3] D. Ionescu and M. Kovaci, "About the negative permittivity of some metamaterial composites-simulational study," in Proceedings of the 17th IEEE International Symposium for Design and Technology of Electronics Packages (SIITME '11), pp. 197200, October 2011.

[4] A. Grbic, "A 2-D composite medium exhibiting broadband negative permittivity and permeability," in Proceedings of the IEEE Antennas and Propagation Society International Symposium, pp. 4133-4136, 2006.

[5] J. B. Pendry, A. J. Holden, W. J. Stewart, and I. Youngs, "Extremely low frequency plasmons in metallic mesostructures," Physical Review Letters, vol. 76, no. 25, pp. 4773-4776, 1996.

[6] J. B. Pendry, A. J. Holden, D. J. Robbins, and W. J. Stewart, "Magnetism from conductors and enhanced nonlinear phenomena," IEEE Transactions on Microwave Theory and Techniques, vol. 47, no. 11, pp. 2075-2084, 1999.

[7] D. R. Smith, W. J. Padilla, D. C. Vier, S. C. Nemat-Nasser, and S. Schultz, "Composite medium with simultaneously negative permeability and permittivity," Physical Review Letters, vol. 84, no. 18, pp. 4184-4187, 2000.

[8] C. A. Balanis, Antenna Theory, John Wiley \& Sons, 1999.

[9] R. W. Ziolkowski, "Design, fabrication, and testing of double negative metamaterials," IEEE Transactions on Antennas and Propagation, vol. 51, no. 7, pp. 1516-1529, 2003.

[10] H. O. Moser, B. D. F. Casse, O. Wilhelmi, and B. T. Saw, “Terahertz response of a microfabricated rod-split-ring-resonator electromagnetic metamaterial," Physical Review Letters, vol. 94, no. 6, Article ID 063901, 2005.

[11] R. W. Ziolkowski, "Design, fabrication, and testing of double negative metamaterials," IEEE Transactions on Antennas and Propagation, vol. 51, no. 7, pp. 1516-1529, 2003.

[12] W. Withayachumnankul and D. Abbott, "Metamaterials in the Terahertz regime," IEEE Photonics Journal, vol. 1, no. 2, pp. 99118, 2009.

[13] Y. Minowa, M. Nagai, H. Tao et al., "Extremely thin metamaterial as slab waveguide at terahertz frequencies," IEEE Transactions on Terahertz Science and Technology, vol. 1, no. 2, pp. 441449, 2011.

[14] C. Sabah, "Tunable metamaterial design composed of triangular split ring resonator and wire strip for S-and C-microwave bands," Progress in Electromagnetics Research B, vol. 22, pp. 341357,2010

[15] C. Sabah and S. Uckun, "Triangular split ring resonator and wire strip to form new metamaterial," in Proceedings of 29th General Assembly of the International Union of Radio Science, Chicago, Ill, USA, August 2008.

[16] H. Benosman and N. B. Hacene, "Design and simulation of double "S" shaped metamaterial," IJCSI International Journal of Computer Science Issues, vol. 9, no. 2, pp. 534-537, 2012. 

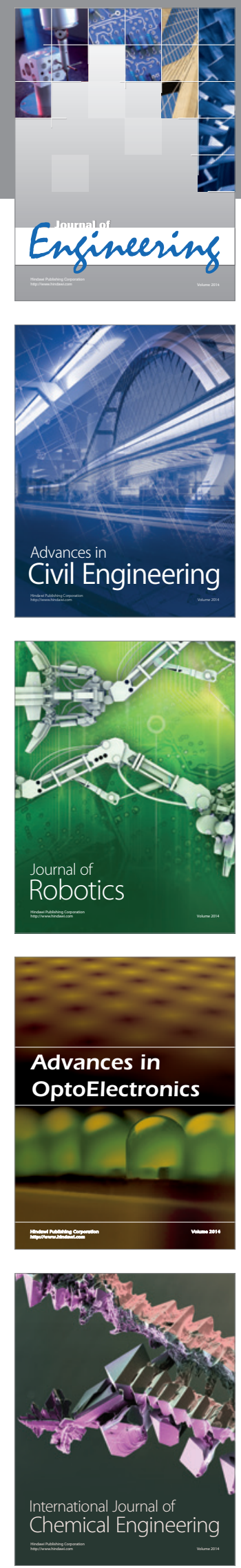

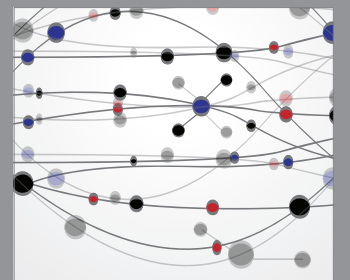

The Scientific World Journal
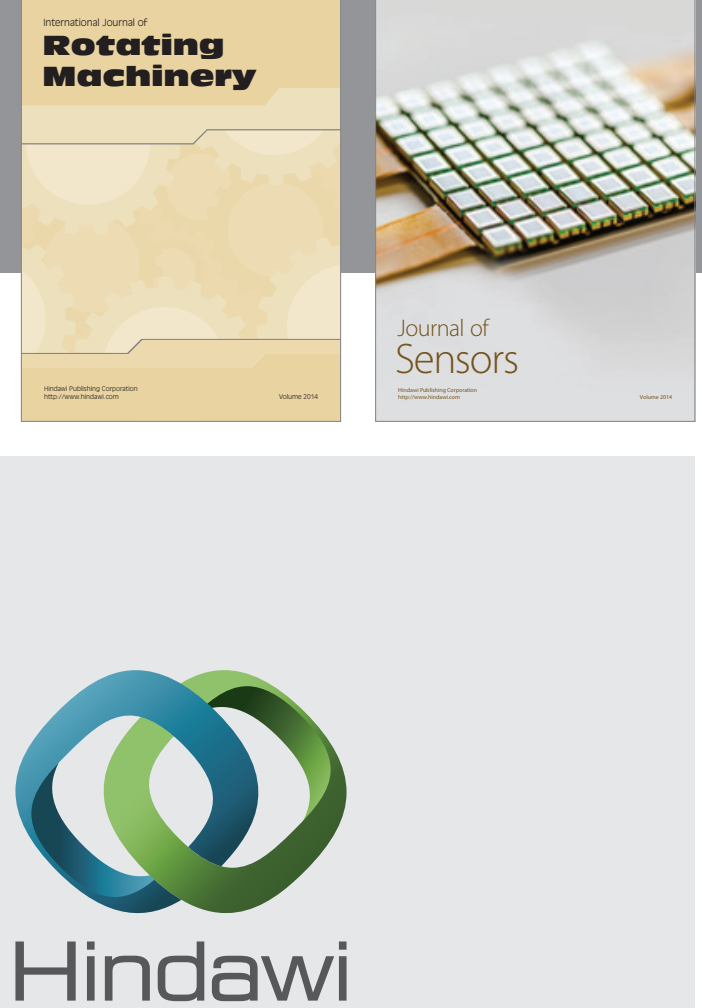

Submit your manuscripts at http://www.hindawi.com
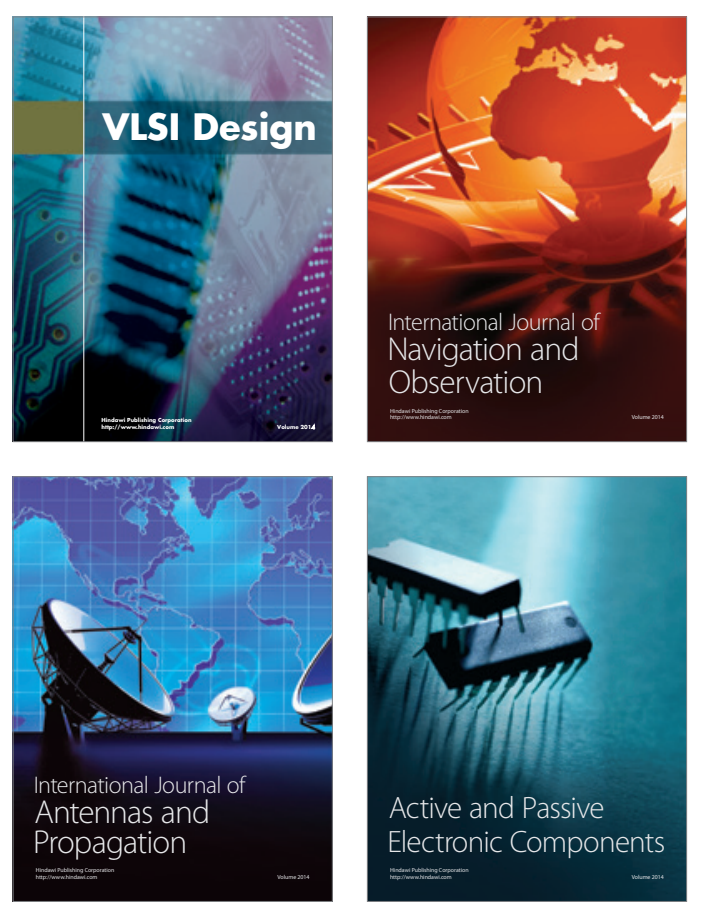
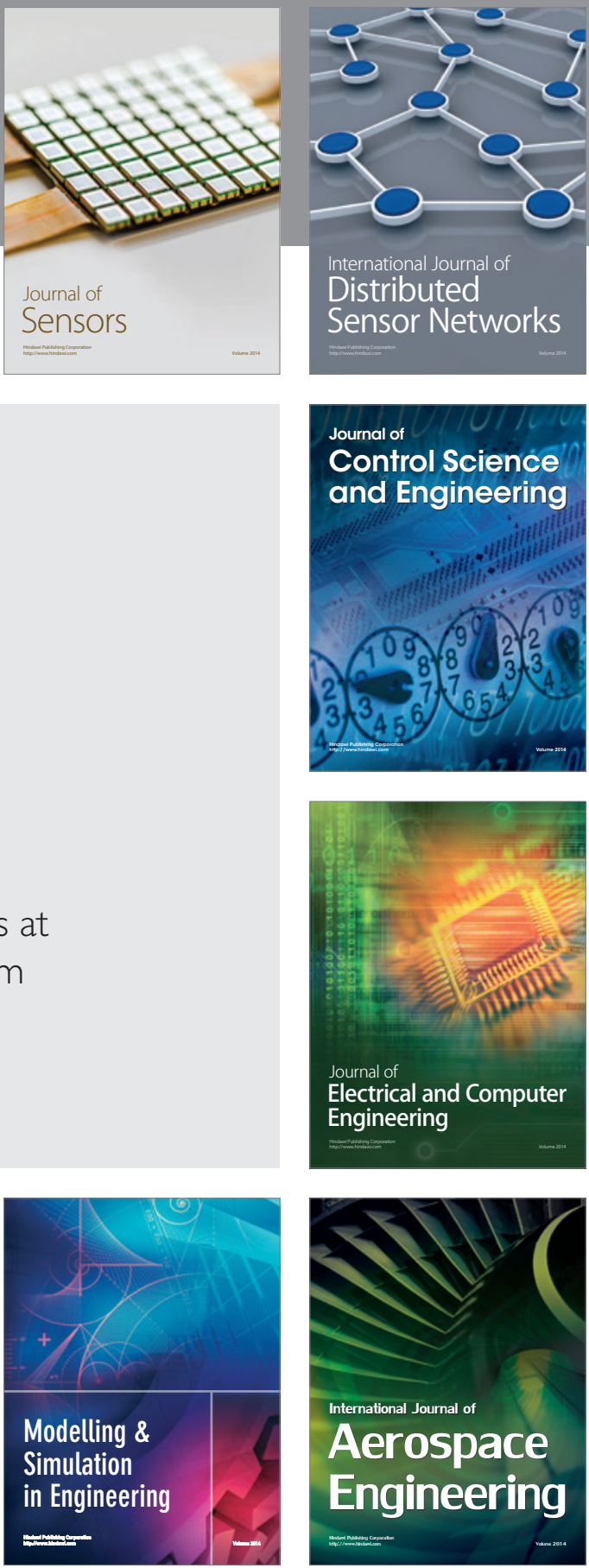

Journal of

Control Science

and Engineering
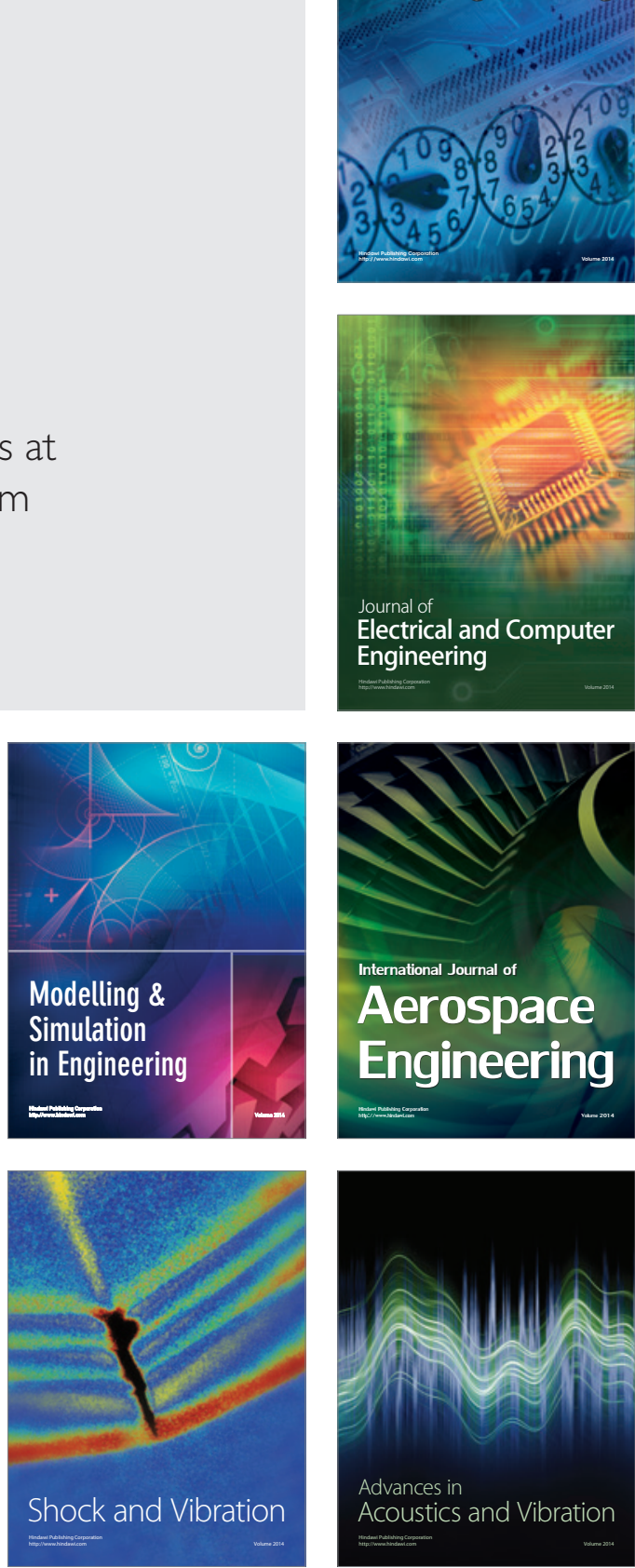